\title{
Cribriform Pattern at the Surgical Margin is Highly Predictive of Biochemical Recurrence in Patients Undergoing Radical Prostatectomy
}

\author{
Kyung Hwan Kim ${ }^{1}$, Ja Yoon $\mathrm{Ku}^{1}$, Chan Ho Lee², Won Young Park ${ }^{3}$, Hong Koo Ha ${ }^{1,4}$ \\ ${ }^{I}$ Department of Urology, Pusan National University Hospital, Pusan National University School of Medicine, Busan, \\ Korea \\ ${ }^{2}$ Department of Urology, Inje University Busan Paik Hospital, Busan, Korea \\ ${ }^{3}$ Department of Pathology, Pusan National University Hospital, Pusan National University School of Medicine, Busan, \\ Korea \\ ${ }^{4}$ Biomedical Research Institute, Pusan National University Hospital, Busan, Korea
}

Objectives: We investigated the relationship between cribriform patterns and biochemical recurrence in patients with positive surgical margins after radical prostatectomy.

Methods: This study was based on radical prostatectomy specimens obtained from 817 patients (165 with margin-positive status) collected at a single center between 2010 and 2016. We retrospectively analyzed and compared body mass index, preoperative prostate-specific antigen, Gleason score, operative methods, postoperative Gleason score, pathological Tstage, tumor percentage involvement, lymphatic and perineural invasion, prostate-specific antigen nadir, location and length of the positive margin, cribriform pattern status, and Gleason grade at the surgical margin in terms of their association with biochemical recurrence. Risk factors for biochemical recurrence were also investigated.

Results: 21\% (31/146) of surgical margin-positive patients had a cribriform pattern. Nadir prostate-specific antigen, perineural invasion and biochemical recurrence rates were significantly higher in cribriform pattern present group than absent group $(P=0.031,0.043$ and 0.045 , respectively). According to the Cox regression model, postoperative Gleason score, tumor percentage involvement, location and length of the positive margin, and the presence of a cribriform pattern at the surgical margin were significant predictive factors of biochemical recurrence $(P=0.022,<0.001,0.015,0.001$, and 0.022 , respectively). Moreover, the biochemical recurrence risk was approximately 3 -fold higher in patients with a cribriform pattern at the surgical margin than in those without (HR: 3.41, 95\% CI 1.20-9.70, $P=0.022$ ).

Conclusions: A cribriform pattern at the surgical margin is a significant predictor of biochemical recurrence in patients who undergo radical prostatectomy.

Key Words: Prostatectomy, Prostate-specific antigen, Prostatic Neoplasms, Recurrence, Surgical margin

Gleason grading is fundamental for making therapeutic decisions and predicting the prognoses of patients with prostate cancer, and is based on the architectural growth pattern of the tumor. This grading system was proposed by Donald F. Gleason in 1966, and has been con-

Corresponding Author: Hong Koo Ha, Department of Urology, Pusan National University Hospital, Pusan National University School of Medicine, 179 Gudeok-Ro, Seo-Gu, Busan 49241, Korea Tel: +82-51-240-7351 Fax: +82-51-247-5443 E-mail: hongkooha@naver.com

Received: Jun. 10, 2019 Revised: Sep. 05, 2019 Accepted: Oct. 15, 2019

\section{(c) (1) (3)}

Articles published in Kosin Medical Journal are open-access, distributed under the terms of the Creative Commons Attribution Non-Commercial License (http://creativecommons.org/licenses/by-nc/4.0/) which permits unrestricted non-commercial use, distribution, and reproduction in any medium, provided the original work is properly cited. 
tinuously modified and revised since. In 2014, the International Society of Urological Pathology (ISUP) Consensus Conference introduced a new Gleason grading system; some of its principal components were that all cribriform glands should be labeled as Gleason grade 4, and that a Gleason score (GS) of 7 should be categorized as grade group 2 or 3 based on a primary Gleason grade of 3 or 4, respectively. ${ }^{1}$ Earlier, the 2005 ISUP Consensus Conference on Gleason Grading of Prostatic Carcinoma considered cribriform glands indicative of a Gleason pattern 3 or 4 depending on the glands' sizes, regularity of the contour, and morphology of the lumina. Cribriform glands were considered Gleason grade 3 when they were small with round lumina and regular contours; a GS of 7 was regarded as a single entity encompassing $3+4$ or $4+3$ patterns. $^{2}$ At the 2014 ISUP Consensus Conference, there were important modifications to the Gleason grading system that highlighted the risk of Gleason grade 4 prostate cancers; these changes were largely attributed to milestone studies that demonstrated unfavorable oncological outcomes in Gleason grade 4 lesions, especially those that exhibited a cribriform pattern (CP). In 2011, Iczkowski et al. found that the presence of $\mathrm{CP}$ in prostate specimens after radical prostatectomy was significantly associated with biochemical recurrence (BCR) regardless of the size of the cribriform glands. ${ }^{3}$ Furthermore, Dong et al. suggested that $\mathrm{CP}$ was a predictive factor of biochemical failure and metastasis after radical prostatectomy, ${ }^{4}$ while Kweldam et al. demonstrated that $\mathrm{CP}$ was an adverse predictive factor for disease-specific survival as well as metastasis-free survival. ${ }^{5,6}$ In this study, we hypothesized that $\mathrm{CP}$ at the surgical margin is a strong risk factor for BCR after prostatectomy. The aim of our current study was to investigate the clinical significance of $\mathrm{CP}$ at the surgical margin in patients who underwent radical prostatectomy.

\section{METRIALS AND METHODS}

\section{Patient enrollment}

We identified 817 consecutive patients who underwent radical prostatectomy between August 1999 and June 2016 at Pusan National University Hospital (PNUH), Busan, South Korea. Of these patients, 165 were found to have positive surgical margins after radical prostatectomy according to their pathological reports (radical retropubic prostatectomy in 11 cases, laparoscopic radical prostatectomy in 129, and robot-assisted laparoscopic radical prostatectomy in 25). The surgical margin CP statuses for 19 patients with positive margins were not clearly indicated in their pathological reports; these patients were therefore excluded from our analysis. The final cohort comprised the remaining 146 patients, 31 of whom had CP. This study was approved by the ethics committee of PNUH (Institutional review board number, H-1905-014-079).

\section{Clinical characteristics}

We obtained clinical data including age at the time of diagnosis (years), body mass index (BMI), preoperative prostate-specific antigen 
(PSA) level $(\mathrm{ng} / \mathrm{mL})$, nadir of PSA level $(\mathrm{ng} / \mathrm{mL})$, surgical method (radical retropubic prostatectomy, laparoscopic radical prostatectomy, or robot-assisted laparoscopic radical prostatectomy), BCR status (present or absent), and time to BCR (months) by reviewing the medical records. Preoperative PSA level was measured via laboratory testing performed during preoperative evaluation. Patients returned for follow-up visits 6 weeks and 3 months after radical prostatectomy, and then every 6 months thereafter. Laboratory tests including PSA levels were conducted serially during the follow-up visits. The PSA nadir was obtained from these office-based serial PSA level measurements. BCR was defined as 2 consecutive PSA level readings of $0.2 \mathrm{ng} / \mathrm{mL}$ or above. BCR-free survival was defined as the time to biochemical recurrence after radical prostatectomy.

\section{Pathological data}

Pathological parameters such as the tumor percentage involvement, preoperative GS, postoperative GS, pathologic T-stage, status of lymph node and perineural invasion, location and length of the positive surgical margin, and CP and GS at the positive margin were documented based on the pathological reports of preoperative biopsy samples as well as postoperative prostatic specimens. All prostate specimens were routinely evaluated at the Department of Pathology of PNUH after they were acquired. A well-experienced uropathologist with pathology board certification, and who was blinded to the patients' information, reviewed all prostatic histologic slides. Pathologic evaluations were performed according to the new Gleason grading system introduced at the 2014 ISUP Consensus Conference. Tumor mapping was used to calculate the tumor percentage involvement. The specimens obtained from radical prostatectomies were sliced and treated as histology slides. The tumor area was evaluated on every slide after being placed on a $1 \mathrm{~mm}^{2}$ background grid. All the tumor areas on these 2-dimensional slides were integrated to estimate the 3-dimensional volume of the tumor. All surgical margins of the prostatic specimens were examined to identify positive surgical margins. Inked edges in these prostatic samples were deemed to be positive surgical margins. The positive surgical margin area was defined according to the tumor location in the specimen: apex, periphery/radius, or base. Furthermore, the length and GS of the positive surgical margins were recorded. The assessment of $\mathrm{CP}$ in prostatic specimens is not routinely performed at our institute; therefore, we consulted the Department of Pathology regarding the presence of $\mathrm{CP}$ at the surgical margin in all the prostatic specimens derived from individuals in our study cohort. The presence of $\mathrm{CP}$ at the surgical margin was documented only when prostatic samples met the definition criteria as introduced by 2014 ISUP Consensus Conference.

\section{Statistical analysis}

We compared clinicopathological factors between specimens with and without $\mathrm{CP}$ at the surgical margin using the Mann-Whitney U-test for continuous variables and Pearson's chi-square 
test for categorical variables. We used Cox proportional hazard models to determine predictive factors for BCR. Survival probabilities were estimated using Kaplan-Meier analysis. The age, PSA level, PSA nadir, surgical method, BCR status, time to BCR, tumor percentage involvement, GS, pathologic T-stage, statuses of lymph node and perineural invasion, location and length of the positive margins, and GS and CP statuses at the surgical margin were subjected to multivariate analysis. All tests in this study were two sided with 5\% significance level. The SPSS Statistics 20 software (IBM, Chicago, IL) was used for all statistical analysis.

\section{RESULTS}

\section{Patient characteristics}

The characteristics of the patients $(n=146)$ are listed in Table 1. The median follow-up period for 146 patients was 37.5 months (interquartile range [IQR] $18.93-62.47$ months). The median follow-up periods for patients with $\mathrm{CP}$ at the surgical margin and for those without were 27.6 (IQR 20.10 - 55.65) and 38.3 (IQR 16.89-62.59) months, respectively $(P=0.58)$. The CP-present and $\mathrm{CP}$-absent groups had similar distributions of age, BMI, preoperative PSA, tumor percentage involvement, positive margin length, preoperative GS, surgical method, postoperative GS, pathologic T-stage, lymphatic invasion, and location of the positive surgical margins $(P>0.05$ for all).

\section{Relationships between CPs and adverse out- comes}

The presence of $\mathrm{CP}$ was evaluated according to 2014 ISUP guidelines. The typical CP present and absent prostate cancer specimen were presented in Figure 1. Small round cribriform glands were identified in the cancer specimen from left peripheral zone (Fig. 1A). The resection margin was involved by prostate cancer. The cancer

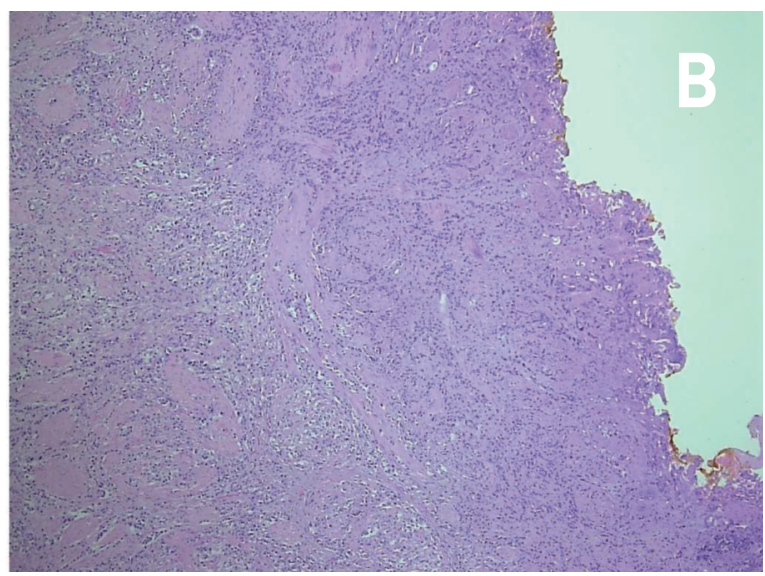

Fig. 1. Pathologic slides of prostate cancer specimen

(A) Prostate cancer specimen of Gleason score $7(4+3)$ showing small and round cribriform glands (cribri form patterns) at positive surgical margin (B) Prostate cancer specimen of Gleason score $9(5+4)$ with infiltrative growth patterns, but not showing crib riform glands 
Table 1. Clinicopathologic characteristics of the patients ( $n=146$ )

\begin{tabular}{|c|c|c|c|}
\hline \multirow[t]{2}{*}{ Factors } & \multicolumn{2}{|c|}{ Cribriform growth pattern } & \multirow[b]{2}{*}{$P$-value } \\
\hline & present $(n=31)$ & absent ( $\mathrm{n}=115$ ) & \\
\hline Age lyears) & $66.0(62.0-70.0) *$ & $68.0(64.0-73.0) *$ & 0.079 \\
\hline Body mass index $\left(\mathrm{kg} / \mathrm{m}^{2}\right)$ & $23.0(21.4-24.6) *$ & $23.0(21.0-25.0) *$ & 0.377 \\
\hline Preoperative PSA (ng/ml) & $15.8(6.2-25.4) *$ & $11.0(4.2-17.8) *$ & 0.689 \\
\hline Tumor \% involvement (\%) & $30.0(6.6-53.4) *$ & $20.0(6.5-33.5) *$ & 0.388 \\
\hline Nadir PSA (ng/ml) & $0.03(0.00-1.11) *$ & $0.01(0.00-0.08) *$ & 0.031 \\
\hline Time to BCR (months) & $14.9(4.5-40.2) *$ & $35.0(7.3-62.8) *$ & 0.096 \\
\hline Positive margin length (mm) & $5.5(3.9-7.1) *$ & $4.0(2.0-6.0) *$ & 0.299 \\
\hline Preoperative Gleason score (n) & & & 0.959 \\
\hline 7 & $19(61.3 \%)$ & $68(59.6 \%)$ & \\
\hline 8 & $9(29.0 \%)$ & $36(31.6 \%)$ & \\
\hline 9 & $3(9.7 \%)$ & $10(8.8 \%)$ & \\
\hline Surgical method (n) & & & 0.139 \\
\hline RRP & $0(0.0 \%)$ & $8(7.0 \%)$ & \\
\hline LRP & $23(74.2 \%)$ & $90(78.3 \%)$ & \\
\hline RARP & $8(25.8 \%)$ & $17(14.8 \%)$ & \\
\hline Postoperative Gleason score (n) & & & $0.226+$ \\
\hline 6 & $0(0.0 \%)$ & $10(8.8 \%)$ & \\
\hline 7 & $18(58.1 \%)$ & $70(61.9 \%)$ & \\
\hline 8 & $5(16.1 \%)$ & $15(13.3 \%)$ & \\
\hline 9 & $8(25.8 \%)$ & $18(15.9 \%)$ & \\
\hline pT stage (n) & & & $0.121 \dagger$ \\
\hline 1 & $0(0.0 \%)$ & $2(1.7 \%)$ & \\
\hline 2 & $11(35.5 \%)$ & $62(53.9 \%)$ & \\
\hline 3 & $20(64.5 \%)$ & $51(44.3 \%)$ & \\
\hline Lymphatic invasion (n) & & & $0.240 \dagger$ \\
\hline present & $6(19.4 \%)$ & $13(11.3 \%)$ & \\
\hline absent & $25(80.6 \%)$ & $102(88.7 \%)$ & \\
\hline Perineural invasion (n) & & & 0.043 \\
\hline present & $29(93.5 \%)$ & $89(77.4 \%)$ & \\
\hline absent & $2(6.5 \%)$ & $26(22.6 \%)$ & \\
\hline $\mathrm{BCR}(\mathrm{n})$ & & & 0.045 \\
\hline present & $19(73.1 \%)$ & $48(51.1 \%)$ & \\
\hline absent & $7(26.9 \%)$ & $46(48.9 \%)$ & \\
\hline Positive margin area (n) & & & 0.163 \\
\hline Apex & $11(35.5 \%)$ & $48(41.7 \%)$ & \\
\hline Peripheral/Radial & $14(45.2 \%)$ & $32(27.8 \%)$ & \\
\hline Base & $6(19.4 \%)$ & $35(30.4 \%)$ & \\
\hline Positive margin Gleason grade (n) & & & $<0.001$ \\
\hline 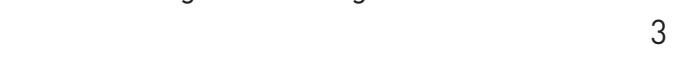 & $0(0.0 \%)$ & $87(75.7 \%)$ & \\
\hline 4 & $31(100.0 \%)$ & $20(17.4 \%)$ & \\
\hline 5 & $0(0.0 \%)$ & 8 (7.0\%) & \\
\hline
\end{tabular}

\footnotetext{
*: Age, body mass index, preoperative PSA, tumor \% involvement, nadir PSA, time to BCR and positive margin length are presented as median values (interquartile range), + : Result of Fisher's exact test. PSA = prostate specific antigen; BCR = biochemical recurrence; RRP = radical retropubic prostatectomy; LRP = laparoscopic radical prostatectomy; RARP = robot-assisted laparoscopic radical prostatectomy.
} 
Table 2. Cox regression for the progression to biochemical recurrence according to cribriform pattern in patient undergone radical prostatectomy

\begin{tabular}{|c|c|c|c|}
\hline \multirow[t]{2}{*}{ Factors } & \multicolumn{3}{|c|}{ Multivariate analysis } \\
\hline & HR & $95 \% \mathrm{Cl}$ & $P$-value \\
\hline Preoperative PSA & 0.99 & $0.97-1.00$ & 0.059 \\
\hline Postoperative Gleason score & & & 0.022 \\
\hline 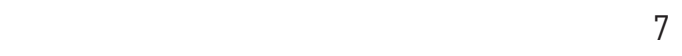 & 8.68 & $1.14-65.97$ & 0.037 \\
\hline 8 & 14.08 & $1.55-127.60$ & 0.019 \\
\hline 9 & 8.62 & $0.98-76.05$ & 0.053 \\
\hline Pathologic T stage & & & 0.096 \\
\hline pT2 stage versus pT1 stage & 0.14 & $0.01-1.40$ & 0.094 \\
\hline pT3 stage versus $\mathrm{pT} 1$ stage & 0.27 & $0.03-2.58$ & 0.253 \\
\hline Tumor \% involvement & 1.03 & $1.02-1.05$ & $<0.001$ \\
\hline Positive surgical margin area & & & 0.015 \\
\hline Peripheral/Radial area & 1.76 & $0.87-3.58$ & 0.117 \\
\hline Apex area & 0.65 & $0.28-1.51$ & 0.315 \\
\hline Cribriform pattern & 3.41 & $1.20-9.70$ & 0.022 \\
\hline Positive surgical margin Gleason grade & & & 0.05 \\
\hline $\mathrm{cos}$ & 0.37 & $0.12-1.15$ & 0.086 \\
\hline 5 & 2.15 & $0.61-7.56$ & 0.235 \\
\hline Positive surgical margin length & 1.1 & $1.00-1.17$ & 0.001 \\
\hline
\end{tabular}

$\mathrm{Cl}=$ confidence interval; PSA = prostate specific antigen

specimen showing infiltrative growth pattern without $\mathrm{CP}$ were demonstrated (Fig. 1B). The Gleason scores were $7(4+3)$ and $9(5+4)$, respectively.

The presence of a $\mathrm{CP}$ at the surgical margin was associated with the nadir PSA level after radical prostatectomy. The median value of the PSA nadir in CP-present patients was higher than that in CP-absent patients ( 0.03 vs. $0.01 \mathrm{ng} / \mathrm{mL}$, respectively, $P=0.031)$. In contrast to lymphatic invasion $(P>0.05)$, perineural invasion was more frequently observed in CP-present patients than in their CP-absent counterparts $(93.5 \%$ vs $77.4 \%$, respectively, $P=0.043$ ).

The proportion of BCR was higher in CP-present patients ( $P=0.045)$; 19 of 26 CP-present patients (73.1\%) and 48 of $94 \mathrm{CP}$-absent patients (51.1\%) experienced BCR. Furthermore, the median time to BCR in patients with CPs at the surgical margins was 14.9 months, which was shorter than that in CP-absent patients (35.0 months).

\section{Risk factors for BCR in margin-positive pa- tients}

We performed multivariate analysis to identify BCR predictors. On multivariate survival analysis using Cox proportional hazard models, postoperative GS, tumor percentage involvement, presence of $\mathrm{CP}$ at the surgical margin, and location and length of the positive surgical margin were predictive factors for BCR in patients with positive surgical margins after radical prostatectomy $(P=0.022,<0.001,0.022,0.015$, and 0.001 , respectively) (Table 2 ). Higher postoper- 


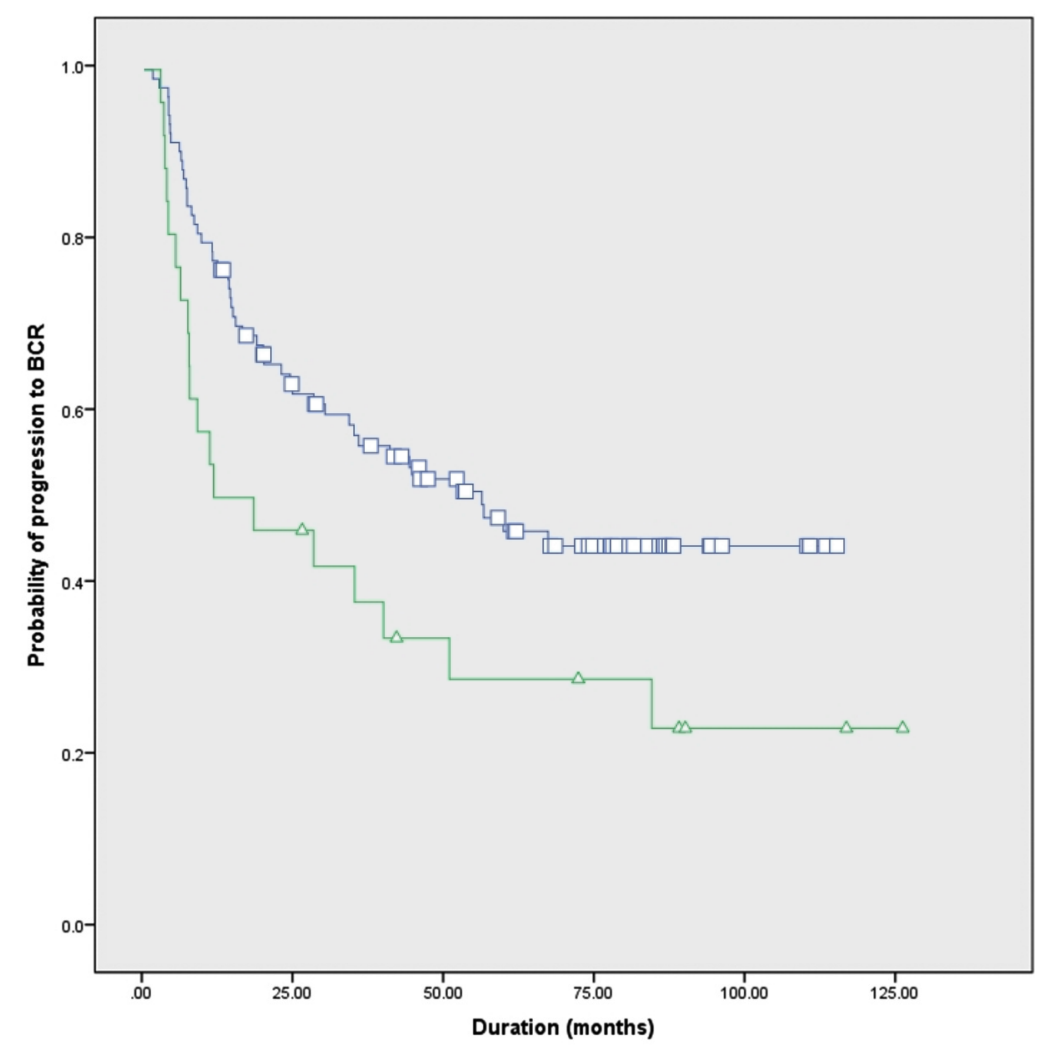

Fig. 2. BCR free survival rate

$B C R=$ biochemical recurrence

$\square$ : absence of cribriform pattern

$\triangle$ : presence of cribriform pattern

log rank $P=0.02$

The median times to BCR in the CP-present and CP-absent

groups were 14.9 (IQR 4.5-40.2) and 35.0 (IQR 7.3-62.8) months, respectively

(log rank $P=0.022$ )

ative GS was associated with a greater risk of BCR; surgical margin-positive patients with GS 7 and GS 8 had approximately 9- and 14-fold higher risks of BCR compared to those with GS 6, respectively (hazard ratios: $8.68[P=0.037]$ and $14.08[P=0.019]$, respectively). Pathologic tumor percentage involvement was also a strong predictive factor for BCR (relative hazard ratio: $1.03, P<0.001)$. The BCR risks differed according to the positive surgical margin locations in the prostatic specimens $(P=0.015)$. The length of the positive surgical margin was also a predic- tive factor for BCR $(P=0.001)$.

\section{Relationship between $\mathrm{CP}$ and BCR}

On multivariate analysis, the presence of $\mathrm{CP}$ at the surgical margin produced a 3-fold higher risk of BCR than CP absence (hazard ratio: 3.41, $P=$ 0.022).

Moreover, the median times to $\mathrm{BCR}$ in the $\mathrm{CP}$ present and CP-absent groups were 14.9 (IQR 4.5-40.2) and 35.0 (IQR 7.3-62.8) months, respectively ( $\log \operatorname{rank} P=0.022$ ) (Fig. 2). BCR occurred approximately 20 months earlier in 
CP-present patients than in their CP-absent counterparts.

\section{DISCUSSION}

$\mathrm{CP}$ is known to be associated with unfavorable oncological outcomes such as BCR, distant metastasis, and death. ${ }^{3,4,6,7}$ In our study, we aimed to identify the predictors for BCR and evaluate BCR-free survival in surgical margin-positive patients.

The presence of $\mathrm{CP}$ was identified as a strong predictor of BCR on multivariate analysis using Cox proportional hazard models, which was consistent with previous CP-related studies. The presence of CP negatively impacted BCR-free survival. The median time to BCR in CP-present group was 14.9 months, which was significantly shorter than that in the CP-absent group (35.0 months). Kweldam et al. identified CP as an adverse predictor of metastasis-free and diseasespecific survival. ${ }^{6,7}$ Our study cohort comprised surgical margin-positive patients who underwent radical prostatectomy, and we showed that the negative effects of $\mathrm{CP}$ on $\mathrm{BCR}$ persist in surgical margin-positive patients.

The PSA nadir has also been shown to be a strong predictor of BCR. Shen et al. suggested that the ultrasensitive nadir PSA predicted the risk of biochemical failure after radical prostatectomy; ${ }^{8}$ patients with a nadir PSA $<0.01 \mathrm{ng} / \mathrm{mL}$ were at low risk for BCR. In our study, the nadir PSA level was not a predictive factor for BCR on multivariate analysis; however, the median nadir PSA level in the CP-absent group was lower than that in the CP-present group. This might be attributed to differences in patient characteristics. In the study by Shen et al., the proportion of surgical margin-negative patients was substantial $(359 / 423$ with a nadir PSA $<0.01$ $\mathrm{ng} / \mathrm{mL}, 64 / 75$ with a nadir PSA of $0.01 \mathrm{ng} / \mathrm{mL}$, $12 / 19$ with a nadir PSA of $0.02 \mathrm{ng} / \mathrm{mL}$, and 17/28 with a nadir PSA of $0.04 \mathrm{ng} / \mathrm{mL}$ or greater). On the other hand, our cohort comprised only of patients with positive surgical margins.

Clinical parameters such as preoperative PSA, clinical tumor stage, GS, and pathological stage were found to be predictive factors for BCR. In other studies, biopsy GS, PSA level, clinical TNM stage, postoperative GS, positive margin length, and organ confinement were predictive of BCR. ${ }^{5,9-13}$ In this study we found that postoperative GS was such a significant predictor of $\mathrm{BCR}$ as $\mathrm{CP}$ on multivariate analysis $(P=0.022)$. Postoperative GS 7 and 8 showed 9 and 14 times higher risk for BCR than postoperative GS 6 .

The length and location of positive surgical margin are known risk factors for BCR after radical prostatectomy. In a study of site-specific surgical margins in 117 patients, Hsu et al. showed that the lengths of the positive surgical margins located in the anterior fibromuscular zone and apex were significantly associated with BCR. ${ }^{10}$ In our study, the proportion of pathologic tumor tissue and the length of positive margin were strong adverse predictors of $\mathrm{BCR}$ (relative hazard ratio = 1.03 and 1.1, respectively; all $P<0.001$ ). And we also showed that the location of positive margin was predictive of BCR $(P=0.015)$. Though 
tumor volume and positive margin length were identified to be more significant for BCR, the relative hazard ratios were only 1.03 and 1.1 , respectively, which were even lower than the relative hazard ratio of $\mathrm{CP}$ (relative hazard ratio $=3.41$ ). So the presence of $\mathrm{CP}$ might be the more effective risk factor on BCR than tumor volume or positive margin length. We identified the positive surgical margin area as a risk factor of BCR, but failed to discover the relative degrees of risk between different positive margin locations.

Histologic features of prostate cancer were known to be important prognostic factor for treatment. The grade for prostate cancer has changed over time. ${ }^{14}$ Some recent studies on $\mathrm{CP}$ of prostate cancer elucidated the association of molecular alterations and adverse oncological outcomes. ${ }^{15,16}$ In this study, we focused on the associations between $\mathrm{BCR}$ ratio and conditions of positive surgical margin, such as presence of $\mathrm{CP}$, lengths and areas of the positive surgical margins and positive surgical margin Gleason grade. Among these variables, presence of $\mathrm{CP}$, lengths and areas of the positive surgical margins were identified as BCR risk factors. And CP showed substantial hazard ratio of 3.41 and proved to be a strong predictor of BCR.

Our retrospective cohort study has some limitations. Patients with positive surgical margins were not randomly distributed into the CP-present and CP-absent groups, as this was not possible. Moreover, this was a single center study comprising only 31 patients in the CP-present group. And the number of $\mathrm{CP}$-absent patients was 115 , which was as much as four times of CP- present cases. The difference between numbers of CP-present and CP-absent patients, could deteriorate the reliability of this study. Lastly, only a single uropathologist with pathology board certification reviewed all the pathologic specimens. There was no chance to correct the potential errors on pathological results.

In conclusion, postoperative Gleason score, tumor percentage involvement, location and length of the positive margin and the presence of $\mathrm{a} \mathrm{CP}$ at the surgical margin were identified as risk factors for BCR in patients with positive surgical margins after radical prostatectomy. The presence of $\mathrm{CP}$ showed higher risk for $\mathrm{BCR}$ than $\mathrm{CP}$ absent cases and proved to be a prominent predictor of BCR after radical prostatectomy in the patients with positive surgical margins.

\section{REFERENCES}

1. Epstein JI, Egevad L, Amin MB, Delahunt B, Srigley JR, Humphrey PA, et al. The 2014 International Society of Urological Pathology (ISUP) Consensus Conference on Gleason Grading of Prostatic Carcinoma: Definition of grading patterns and proposal for a new grading system. Am J Surg Pathol 2016;40:244-52.

2. Epstein JI, Allsbrook WC Jr, Amin MB, Egevad LL, ISUP Grading Committee. The 2005 International Society of Urological Pathology (ISUP) Consensus Conference on Gleason Grading of Prostatic Carcinoma. Am J Surg Pathol 2005;29:1228-42. 
3. Iczkowski KA, Torkko KC, Kotnis GR, Wilson RS, Huang W, Wheeler TM, et al. Digital quantification of five high-grade prostate cancer patterns, including the cribriform pattern, and their association with adverse outcome. Am J Clin Pathol 2011;136:98-107.

4. Dong F, Yang P, Wang C, Wu S, Xiao Y, McDougal WS, et al. Architectural heterogeneity and cribriform pattern predict adverse clinical outcome for Gleason grade 4 prostatic adenocarcinoma. Am J Surg Pathol 2013;37:1855-61.

5. Kweldam CF, Kümmerlin IP, Nieboer D, Verhoef EI, Steyerberg EW, Incrocci L, et al. Prostate cancer outcomes of men with biopsy Gleason score 6 and 7 without cribriform or intraductal carcinoma. Eur J Cancer 2016;66:26-33.

6. Kweldam CF, Wildhagen MF, Steyerberg EW, Bangma $\mathrm{CH}$, van der Kwast TH, van Leenders GJ. Cribriform growth is highly predictive for postoperative metastasis and disease-specific death in Gleason score 7 prostate cancer. Mod Pathol 2015;28:45764.

7. Kweldam CF, Kümmerlin IP, Nieboer D, Verhoef EI, Steyerberg EW, van der Kwast $\mathrm{TH}$, et al. Disease-specific survival of patients with invasive cribriform and intraductal prostate cancer at diagnostic biopsy. Mod Pathol 2016;29:630-6.

8. Shen S, Lepor H, Yaffee R, Taneja SS. U1trasensitive serum prostate specific antigen nadir accurately predicts the risk of early relapse after radical prostatectomy. J Urol
2005;173:777-80.

9. Song C, Kang T, Yoo S, Jeong IG, Ro JY, Hong JH, et al. Tumor volume, surgical margin, and the risk of biochemical recurrence in men with organ-confined prostate cancer. Urol Oncol 2013;31:168-74.

10. Hsu M, Chang SL, Ferrari M, Nolley R, Presti JC Jr, Brooks JD. Length of site-specific positive surgical margins as a risk factor for biochemical recurrence following radical prostatectomy. Int J Urol 2011;18:272-9.

11. Han M, Partin AW, Zahurak M, Piantadosi S, Epstein JI, Walsh PC. Biochemical (prostate specific antigen) recurrence probability following radical prostatectomy for clinically localized prostate cancer. J Urol 2003;169:517-23.

12. D'Amico AV, Whittington R, Malkowicz SB, Schultz D, Blank K, Broderick GA, et al. Biochemical outcome after radical prostatectomy, external beam radiation therapy, or interstitial radiation therapy for clinically localized prostate cancer. JAMA 1998;280:96 9-74.

13. Kinoshita H, Shimizu Y, Mizowaki T, Takayama K, Norihisa Y, Kamoto T, et al. Risk factors predicting the outcome of salvage radiotherapy in patients with biochemical recurrence after radical prostatectomy. Int J Urol 2013;20:806-12.

14. McKenney JK. The present and future of prostate cancer histopathology. Curr Opin Urol 2017;27:464-8.

15. Truong M, Frye T, Messing E, Miyamoto H. Historical and contemporary perspectives 
on cribriform morphology in prostate cancer.

Nat Rev Urol 2018;15:475-82.

16. Kweldam CF, van der Kwast, van Leenders

GJ. On cribriform prostate cancer. Transl

Androl Urol 2018;7:145-54. 